\title{
Comparison of broadband intelligent network signalling architectures
}

\author{
Olli Martikainena, Tapani Karttunen $b$, Valeri Naoumovc, \\ Konstantin Samouylovd \\ aTelecom Finland, Helsinki \\ 'Lappeenranta University of Technology, Lappeenranta \\ cInstitute for Problems of Information Transmission, \\ Russian Academy of Sciences, Moscow \\ ${ }^{d}$ Russian Peoples' Friendship University, Moscow
}

\begin{abstract}
In this paper we compare different distributed intelligent network signalling architectures based on broadband transmission and switching. As a result of the comparison we choose one example architecture, where we specify the broadband equivalents of Service Switching Point $(B-S S P)$ and Service Control Point $(B-S C P)$. The protocols for user signalling and broadband application part as well as the service distribution architecture are discussed. With the proposed Broadband Intelligent Network Signalling Architecture an example interactive multimedia service is concepted and analyzed.
\end{abstract}

\section{INTRODUCTION}

In modern telecommunications deeply influential changes are taking place, caused by the emerging competitive telecommunications services market and the new technological breakthroughs. The market changes are due to the integration of telecommunications and information technology, which makes it possible to develop data intensive telecommunications services. There are two types of application data involved: the customer and service management data, and service content data. Typical examples of the former are the mobile and value added network (VANS) services, where customer location data and customer personal service data are used for network control. With broadband networks media service applications bring interactive real time video and multimedia contents available to users. Examples of media services are digital interactive TV, video on demand services (VOD), electronic press and publishing. The service portfolio will move from Interconnection services towards Mobile, VANS and Media services (Figure 1-1). 


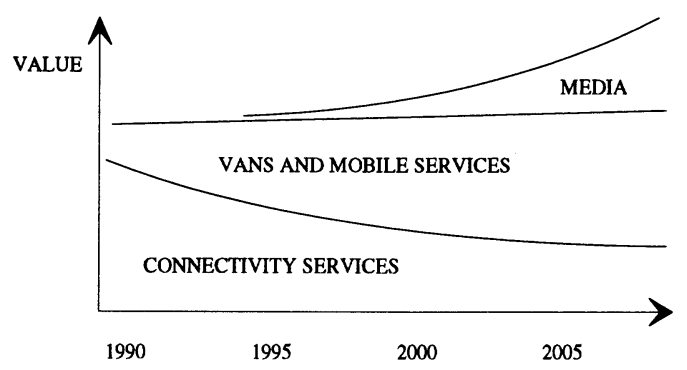

Figure 1-1: Service Portfolio Chances

The technological requirements for these new services are cost effective broadband transmission and access technologies, flexible computer based management and control of networks, switching and service applications and the support of mobility.

In technology substantial new breakthroughs are going on. The introduction of cellular radio networks and mobility is probably the most influential one in the next few years. The broadband transmission and switching technology is also maturing and will provide a cost effective platform for service provision. When the broadband customer access will be available, interactive business and consumer services based on video and multimedia will become possible. Common to all these developments will be the computer controlled structure of modern telecommunications, where protocols, application technology and resource management are key factors.

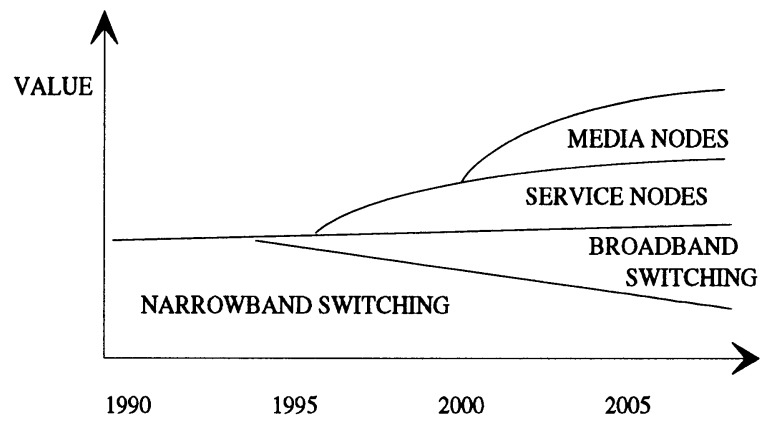

Figure 1-2: Switching and Service Nodes

In modern telephony the most promising computer control and management concept is the Intelligent Network $(I N)$. It facilitates the development and management of new services by service providers. Intelligent networks can be considered as the bridging technology between the customer based service management, the service application execution and the connection control in the networks. Considering the mobile and broadband technologies it is a natural question, how this concept of intelligence will develop with these new networks. It seems to be clear that the service applications will be distributed over terminals and different service control and media provider nodes (Figure 1-2). Examples of distributed intelligence can be 
found from Internet World Wide Web $(W W W)$ and the specifications of Universal Mobile Telecommunications Services (UMTS).

\section{MOTIVATION FOR DISTRIBUTED INTELLIGENCE}

The new broadband and mobile infratructures will enable new service types and more versatile roles for service providers. We believe that in broadband and media services the integrated service combinations will grow more important. This is because there are advantages of scale in basic interconnection and basic media production. Hence the basic production has to be done in a large scale, preferably by global network or service providers. On the other hand, the specialized knowhow depending on segmented markets creates specialized enterprises, that use their expertise locally or even in global scale. The generic and specialized services considered as product elements can be combined to synergic concepts [ValC]. The key question is the ability to exploit strong synergies of different basic and specialized product elements so that the customer perceived value can be maximized (Figure 2-1).

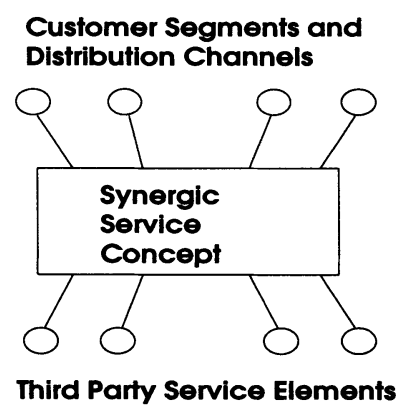

Figure 2-1. Service product consisting of several elements

To facilitate the provision of new services there are demands for open service environments and common service architectures. How these demands can be taken into consideration in the evolution of Intelligent Networks is an important question. Let us first consider the demands in more detail.

\subsection{New Service Types}

The Intelligent Network Architecture (INA) enables rapid development and deployment of advanced services on the current telephony network. Its main principle is the separation of the supplementary services control aspects from the connection control aspects. When the broadband transmission, switching and access capabilities become available in the basic network, the scope and scale of supplementary services will grow remarkably. Different supplementary service types as well as different service provider roles will be present. The following service types can be identified:

- Voice and media services provided in special service nodes by network operators or service providers. 
- Intelligent network services provided in intelligent switches or separate service nodes usually by network operators.

- Mobility services provided today in intelligent switches by mobile network operators.

Voice and media services are controlled by the user with signalling sequences and the service data is resident in databases, which should be interfaced to the network. A promising user and service signalling system for them is the ISDN D-channel signalling, which is separated from the ISDN B-channels. Intelligent Network architecture separates the connection control and service logic. However, the narrowband IN user signalling is still the basic telephony signalling, which is in the speech channel in the access network. In mobile services signalling must be separated from calls because roaming and handovers take place independently of calls. Hence in GSM and DECT the ISDN type signalling protocols have been developed.

\subsection{Different Roles of Enterprises}

In telecommunications the enterprises can be categorized as different stakeholders with respect to the following roles:

- Service users and service subscribers which use the network and service capabilities.

- Network provider that provides the access, transport and switching capacity.

- Service provider that providers services using the network interconnectivity.

- Service manager that manages the operation of services and maintains the normal condition of the network.

- Service broker that subscribes to the service and provides it to another subscriber.

- Others such as network and service designers.

We would like to introduce also the following role:

- Concept provider that integrates different services as synergic combinations and provides these combinations for the service users. Concept provider works in an enterprise network using other service providers as subcontractors and network providers as distribution channels.

How the service logic programs are distributed and interfaced with respect to the different roles is a question that has to be studied.

\subsection{Service Architectures}

Since the service types and the organizational roles of service providers will be more fragmented, the management and interworking of networks and services should be quaranteed. For this purpose unified service architectures will be needed. There are several standardization and research inputs for these kinds of architectures, such as:

- The ISDN Supplementary Services Framework [Q932], where call and supplementary service control messages can be separated from the actual data stream. A lot of pioneering work for supplementary services specification has been done in ISDN recommendations [Q950]. 
- IN, Intelligent Network architecture [Q1200], which specifies the IN Service Processing Model separating call control and service logic, and the INCM Conceptual Model for service specification and distribution for current telephony networks.

- ROSA, the RACE Open Service Architecture [R1093], which defines a set of concepts and rules for building services that are open in time, space and technology. The ROSA conceptual model divides a service in terms of access, core, management and transport capabilities.

- CASSIOPEIA is a RACE project which has continued the ROSA work in defining an Open Services Architecture (OSA).

- INA, the Bellcore Information Network Architecture [Bellc], which separates the architecture into user, data and processing parts and classifies the service management functions into fault, configuration, accounting, performance and security management.

- ODP, the ISO work on Open Distributed Processing [N7524] defines a reference architecture for distributed systems using the enterprise, information, computation, engineering and technology viewpoints and associated languages.

- TINA, Telecommunications Information Networking Architecture [TINA], is a service architecture defined by TINA-C consortium. TINA has adopted ODP reference architecture and applies Object Modelling Technique [OMT], GDMO [X722] and General Relationship Model [X725] specifications in modelling the information concepts. The computation modelling concepts are based on structures and interactions between computational objects, such as operational and stream interfaces, traders and servers. The engineering modelling concepts are based on Distributed Processing Environment (DPE) infrastructure, that provides distribution transparencies by kernel, server and stub -parts.

In ATM networks the control signalling can be independent of the connections, as in ISDN networks, which gives new possibilities in the introduction of intelligence. Since the Intelligent Network Architecture is dominant in the present day networks, there is a clear need to analyse its possible developments with emerging broadband and mobile networks. In this study also the different roles of service users and providers are to be taken into account.

\section{SWITCHING AND INTELLIGENCE MODELLING}

The IN Service Processing Model is designed for modularization of the network functions and standardization of the communication interfaces between network functions [Q1201].

The three main elements of the Service Processing Model are the basic call processes, the "hooks" that allow the basic call processes to interact with IN service logic, and IN service logic that can be "programmed" to implement new supplementary services (Figure 3-1).

The main principles of the Service Processing Model are:

- The basic call process is available all over the network and is designed to support, with optimal performance, network services that do not require special features.

- "Hooks" are to be added to the basic call process forming the links to the service logic in the Service Switching Points (SSP). The "hooks" are trigger points that are able to start an interaction session with the IN service logic. For this it should continuously check the basic call process for the occurrence of conditions on which an interaction session with IN service logic should be started. During an interaction session the basic call process can be temporarily suspended. 
- IN service logic uses a programmable software environment in Service Control Points $(S C P)$. New supplementary services can be created by means of "programs" containing IN service logic. The IN service logic is able, via the "hooks", to interact with the basic call process. In this way IN service logic can control the basic call process.

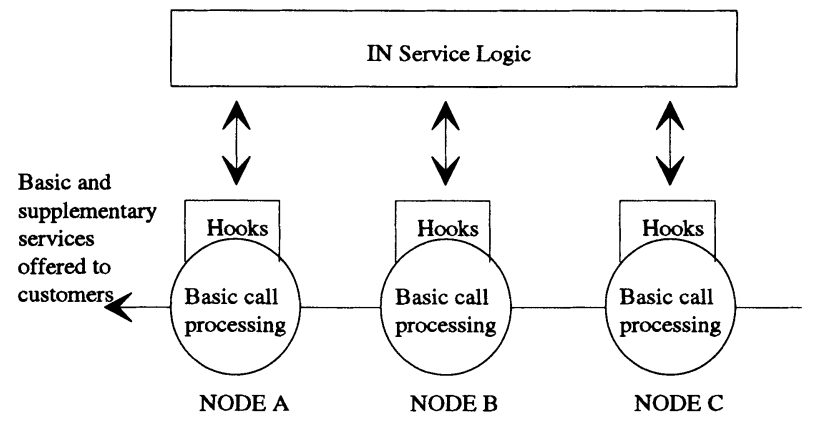

Figure 3-1: IN Service Processing Model

Thus, by changing logic at the service control point and modifying network data, a new service that uses existing network capabilities can readily be implemented.

In addition $\mathbb{I N}$ service logic can decide to terminate an interaction session with the basic call process. The basic call process will then resume its execution as specified by the IN service logic. In order to allow fast service implementation, the IN service logic should have a logical view of the network resources that constitute the basic call process and additional (specialized) network functions.

In present day implementations the service logic is usually centralized and controls the centralized Service Switching Point (Figure 3-2).

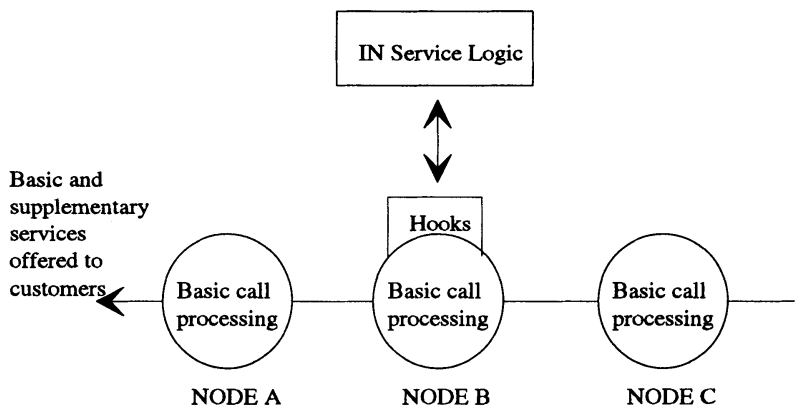

- $\quad$ Figure 3-2: Centralized Service Logic

If the service logic is distributed so that its different parts reside in different network nodes, one has to specify the relevant service logic and network node types. Let us here choose the nodes according to the following roles (Figure 3-3):

- Local Node $(L N)$ used by the Service Subscriber

- Switch operated by the Network Operator 
- Control of Service Combinations operated by Concept Provider

- Service Node ( $S N)$ operated by the Service Provider.

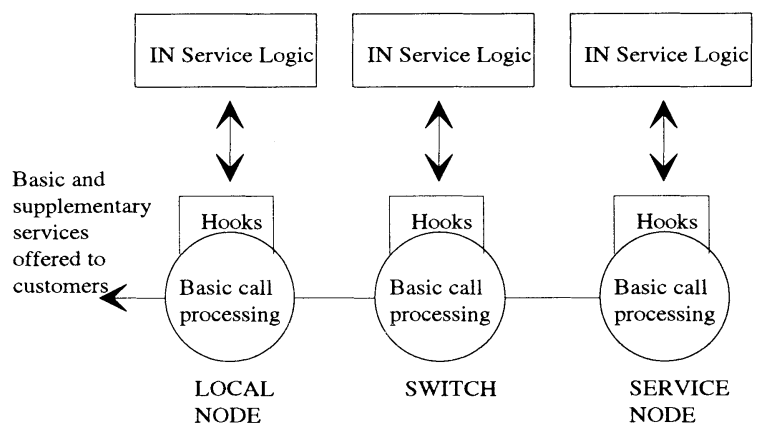

Figure 3-3: Distributed Service Logic

\section{ATM SWITCHING}

\subsection{ATM Networks}

A simplified example for the structure of an ATM network is shown in Figure 4-1. The

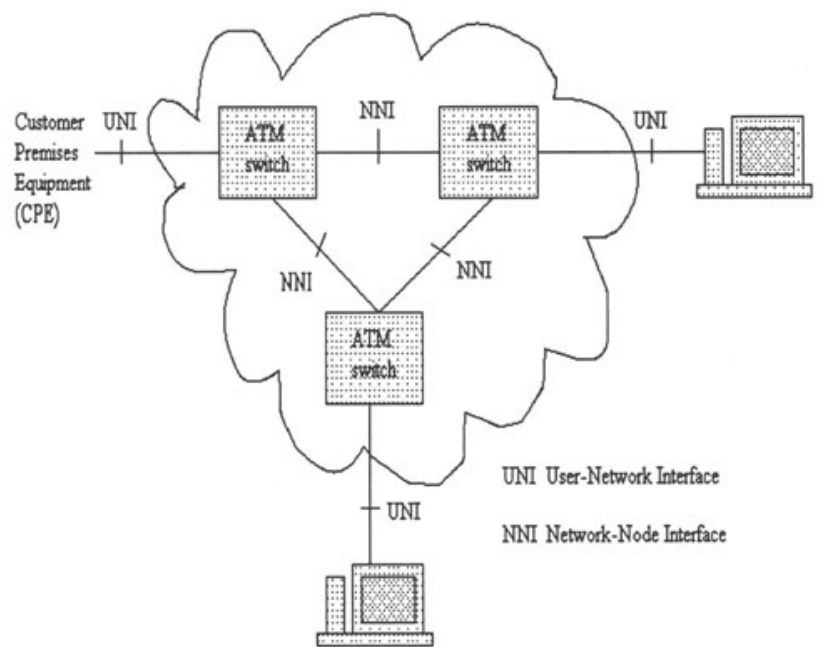

Figure 4-1. ATM network architecture.

various UNI (User-to-Network Interface) and NNI (Network-to-Node Interface) connections could be carried via different physical media, such as the existing Plesiochronous Digital 
Hierarchy $(P D H)$ or the new Synchronous Digital Hierarchy $(S D H)$. Several standards have been defined on how to interface the physical layers, and work is continuing to specify additional physical layers to be used to transport ATM cells. [Forum]

\subsection{Virtual Channels and Virtual Paths}

The concepts Virtual Channel $(V C)$ and Virtual Path $(V P)$ are applied when ATM cells are transported through the entire network (Figure 4-2). Virtual Channel Connections (VCCs) are set up between any source and any destination in the ATM network, regardless of the way it is being routed across the network. Fundamentally, ATM is a connection-oriented technology. The way the network sets up the connection is therefore by means of signalling, i.e. by transmitting a SETUP request which passes across the network to the destination. If the destination agrees to form a connection, the VCC is set up between the two end-systems. A mapping is defined between the Virtual Channel Identifiers $(V C I) /$ Virtual Path Identifiers $(V P I)$ of both UNIs, and between the appropriate input link and the corresponding output link of all intermediate switches. [Forum]

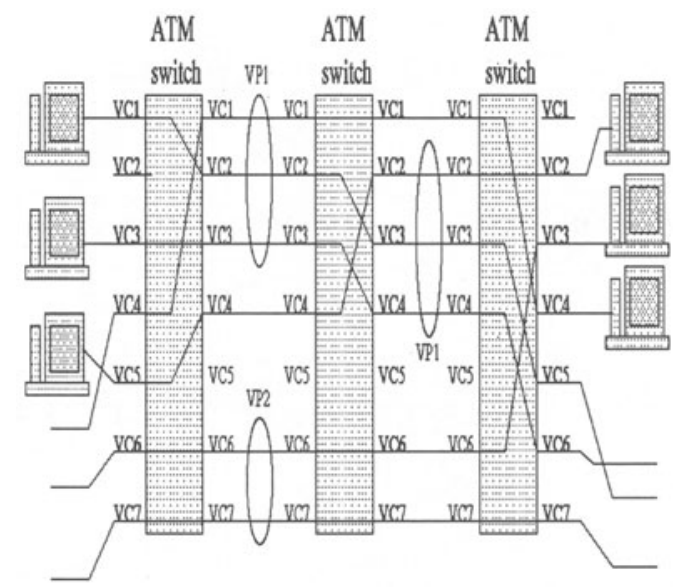

Figure 4-2. Virtual Channels and Virtual Paths.

A VCC is a connection between two communicating ATM end entities, possibly consisting of a concatenation of several ATM VC links. All communication proceeds along this same VCC which preserves cell sequence and provides a certain Quality Of Service (QOS). The Virtual Channel Identifier in the ATM cell header is assigned per network entity-to-entity link, and may change across the network within the same VCC. Each connection is identified by VCC and Virtual Path Connection Identifier (VPCI) consisting of VPI and InterfaceID.

\subsection{ATM Signalling}

The user signalling for call control is specified in [Q2931] for UNI. For point-to-point access configurations $\mathrm{VCI}=5$ is used as the Signalling Virtual Channel (SVC). In Q.2931 the 
following message types for call and connection control are defined: ALERTING, CALL PROCEEDING, CONNECT, CONNECT ACKNOWLEDGE, SETUP, RELEASE, RELEASE COMPLETE, NOTIFY, STATUS and STATUS ENQUIRY.

In B-ISDN there are no additional signalling message types yet to support other features. In ISDN the additional message types to Q.931 defined in Q.932 are: HOLD, HOLD ACKNOWLEDGE, HOLD, REJECT, RETRIEVE, RETRIEVE ACKNOWLEDGE, RETRIEVE REJECT for call related control, and FACILITY and REGISTER for non call related control [Q931, Q932]. The facility messages can convey service dependent information.

Since the SVC is a special VC, the call and facility control type messages can also be used in other VCs. So, they can be used also to control other services than the basic switching. However, these messages may not be sufficient for advanced service control such as the INAP operations between SCP and SSP.

\section{DISTRIBUTION OF INTELLIGENCE IN BROADBAND NETWORKS}

The enhanced broadband network services will be networked applications and the implementations may include several nodes external to the transmission network. If several applications control the service, there is a need of additional functions as compared to the basic call control.

The networked applications may consist of the following: Users that have their own service profiles (own control data), Network that provides the routing and QoS and the Service Providers which offer services and have their own rules how to use the services. Synergic combination of services, called as Service Palettes, can be differentiated with respect to QoS, service types and charging rules.

The ATM switching control is evolving towards ISDN type signalling [Q2931]. Its specification has broadband specific extensions concerning mainly the reservation of the virtual channels and the compatibility with narrowband ISDN. The networked services put new requirements for the protocols to be used. When the services may be controlled from several points, the specified signalling may not be capable enough to deliver control information from one node to another. How Q.2931 and the related protocols could be utilised for this purpose?

Another question is, how to organise service control into network in such way, that the upcoming needs of new services can be fulfilled in a sufficiently flexible way.

\subsection{Control in Existing Systems}

To be able to specify signalling architectures satisfying the demands for distributed intelligence it is helpful to analyse example services. Three existing services could be analysed for this purpose: Mobility services, Intelligent Network services and Media services.

The mobility services are seen as user having portable communication facilities. An example of this is the mobile phone solutions (GSM, DECT). The control of mobility services is handled near the Service Subscriber in terminals and in access networks.

The media services are offered from media service nodes by Service Providers. These MS's in turn have large databases containing the programs or multimedia material. The rules to 
access and to manipulate this information are applied in the server. In other words, the control is placed near the Service Provider.

In traditional Intelligent Network the service is controlled inside the network in Service Control Points. The IN control is focused to control network resources The user or service provider control is more or less added into the concept in a proprietary way.

\subsection{Example Service in Broadband Network}

We shall use the Video on demand (VOD) service as a common example describing the need for broadband network services. VOD service offers television programs and movies to the customers at the time they want to. The main difference to ordinary TV broadcasting is that the service is offered to one customer (unicasting).

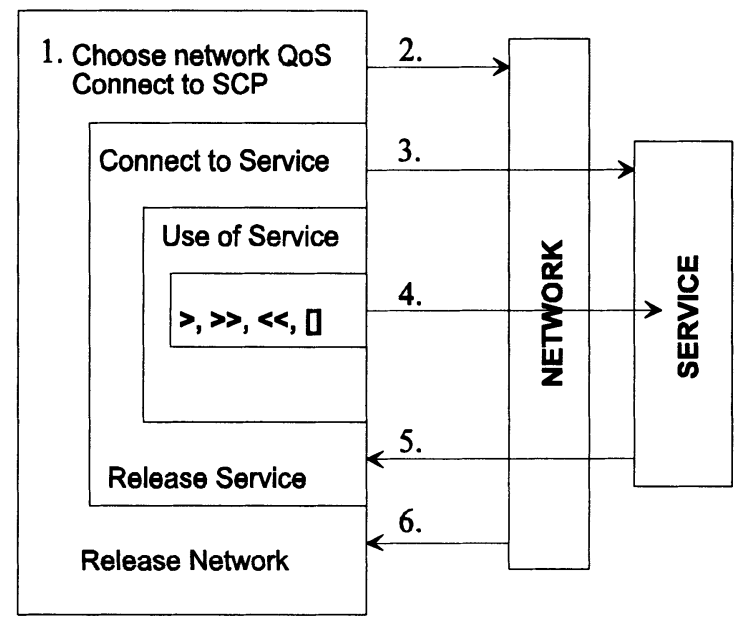

Figure 5-1. VOD Service Phases

The VOD service implementation in broadband networks (on ATM) could be the following (Figure 5-1):

1. the service is selected with service identifier (service number),

2. the service identifier is recognised by the network, the routing and QoS information is retrieved,

3. user is connected to video server,

4. user is controlling video server by the controls offered by server,

5. user is disconnected from video server and

6. network releases the network resources.

\subsection{Analysis of VOD Service Control}

The VOD service presented in previous chapter can be implemented in a real network at least in four different ways: complete control in subscriber terminal, complete control in network 
controller, complete control in video server and the control is distributed among these three instances. Let us analyse these different approaches.

\subsubsection{Subscriber Terminal Based Service Control}

The VOD service control could be placed to the subscriber terminal. In this scenario subscriber terminal (LN, local node) takes care each new connection establishment. The control of the networked service (e.g. how to utilise different connections) is in the user application. In Figure 5-2 the call phases are: a) the original call setup is placed on $\mathrm{VCI}=5$ to connect $\mathrm{SN} \mathrm{1,} \mathrm{b)} \mathrm{the} \mathrm{1st} \mathrm{connection} \mathrm{is} \mathrm{created} \mathrm{to} \mathrm{SN} \mathrm{1,} \mathrm{c)} \mathrm{the} \mathrm{second} \mathrm{call} \mathrm{setup} \mathrm{is} \mathrm{placed} \mathrm{on}$ $\mathrm{VCI}=5$ to connect $\mathrm{SN} 2$ and d) the 2nd connection is created to SN 2.

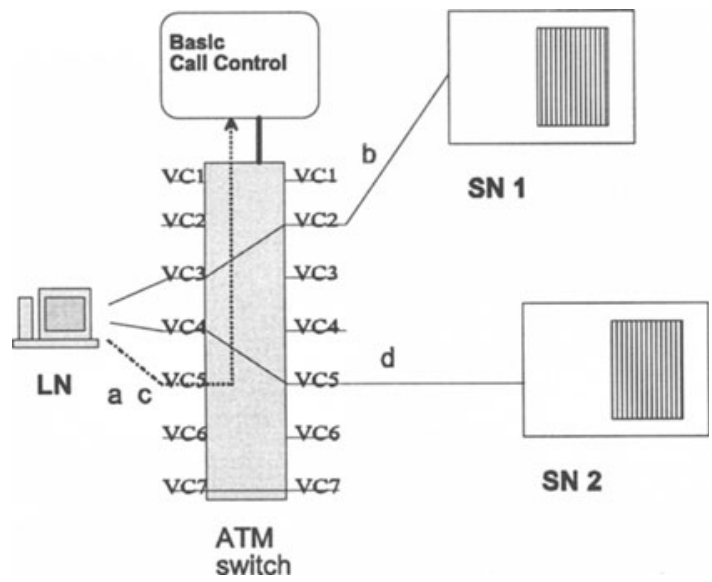

Figure 5-2. The LN based service control

The following consequences can be pointed out:

- the terminal software will be service and furthermore VOD server specific,

- the terminal software will be complex and therefore will rise the cost of the terminal,

- network resource control may be difficult to arrange and

- terminal based security is vulnerable.

This signalling architecture may be optimal for limited service types. It is difficult to support service element or equipment reuse in this architecture.

\subsubsection{Network Based Service Control}

In the next alternative the VOD service control is in the network (either in the switch or in the centralised Service Control Point) (Figure 5-3). In this scenario network controller takes care of each new connection establisment. The control of the networked service, e.g. how to utilise different connections, is in the network control application. The connection creation phases are: a) the original call setup is placed on $\mathrm{VCI}=5$ to connect $\mathrm{SN}, \mathrm{b}$ ) the destination is consulted from SCP, c) SCP responds the routing address, d) the connection leg is created to LN and e) the connection is continued to SN. The possible followup can be created into SCP, when the 
first connection is dropped by SN. $\mathrm{LN}$ is consulted for next operation (e.g. the next $\mathrm{SN}$ address).

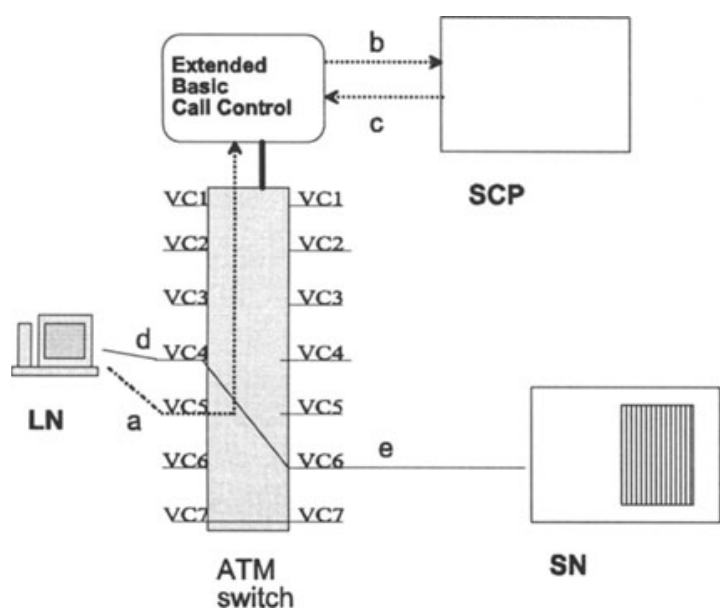

Figure 5-3. The network based service control. The configuration includes SCP. The corresponding scenario could be implemented also with plain switch with its own logic.

The following can be determined from this centralised architecture:

- the service control traffic may cause severe problems in centralised control node,

- service provider specific applications may be difficult to implement,

- service provider and user specific management may be difficult to arrange.

- complex software in centralised control point and

- applications in centralised systems are typically proprietary.

This scenario is similar to the existing Intelligent Network solutions. When looking the existing solutions critically, the efficient service creation has not become true and the external service provider applications cannot be implemented. Furthermore, the management solutions are inflexible and they usually cannot be offered outside network providers due to security problems.

\subsubsection{VOD Server Based Service Control}

The VOD service control can also be placed in the Service Node. In this scenario VOD server takes care each new connection establisment. The server routes forward each new needed connection. This can be seen from the phases in Figure 5-4: a) original call setup is placed by $\mathrm{LN}, \mathrm{b}$ )1st connection is established to SN 1, c) SN 1 requests new connection to SN 2 and d) route to $S N 2$ is established. In this scenario $S N 1$ routes the communication between $L N$ and SN 2. 


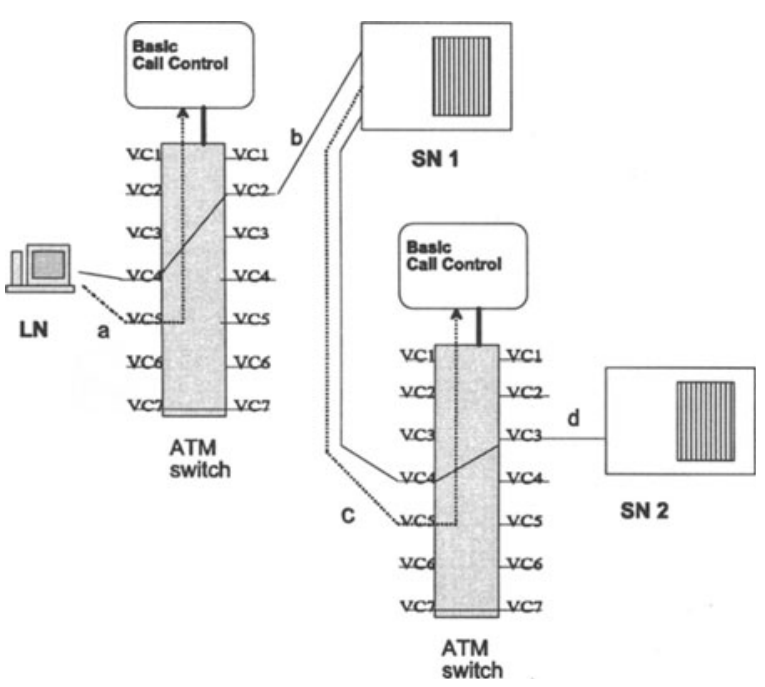

Figure 5-4. The service node (SN) has control of the service. The new connection is routed by original server.

The following can be determined from this scenario:

- the control software will be VOD server and terminal specific,

- the terminal may not be of general purpose,

- inefficient use of network resources may result and

- network QoS cannot be set dynamically.

Basically the VOD service itself is controlled in optimal way in this scenario (server has the control). Anyhow, the network reources cannot be configured dynamically and this causes inefficiency in the network. Also the user profiles are difficult to manage in a VOD server and the service management can control only this special service.

\subsubsection{Distributed Control Between User Terminal, Network and VOD Server}

The control can be distributed in several ways, we present two of the most obvious ones.

1. The external controller has an external control access (separate protocol like CS-1 INAP [Q1218)) (Figure 5-5) or

2. An external controller has an extended D-channel signalling (extension of DSS2 [Q932]) (Figure 5-6).

In both cases several controlling points can be added in the network and these Service Nodes (SN) can control the VC creation. 


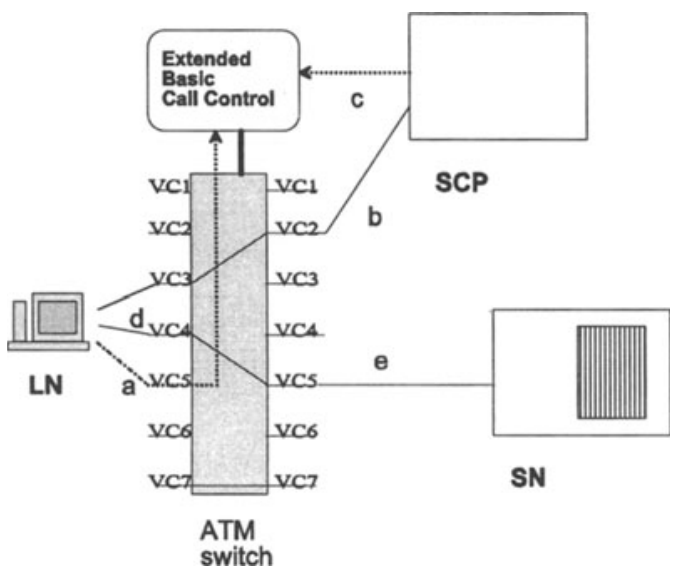

Figure 5-5. The service is distributed. The service access is controlled by SCP and the service itself is controlled by both LN and SN (scenario 1).

The scenario 1 describes the usage of external controller with external control access. The call establishment phases are: a) original call setup to SCP, b) call is established to SCP, SCP is requested to connect the service, c) SCP commands through command interface the switch to establish connection between $\mathrm{LN}$ and $\mathrm{SN}$, and finally d) and e) connection legs are established for a service.

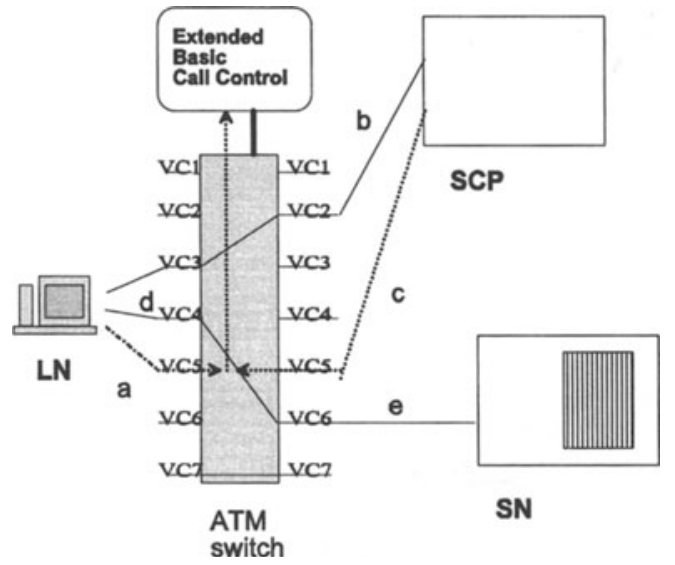

Figure 5-6. The service is distributed. The service access is controlled by SCP and service itself is controlled by both LN and SN. The extension of Q.2931 is utilised as external command channel (scenario 2).

The scenario 2 describes the usage of external controller with Q.2931 extension access. The call establishment phases are: a) original call setup to SCP, b) call is established to SCP, SCP is requested to connect the service, c) SCP commands through Q.2931 interface the 
switch to establish connection between LN and SN, and finally d) and e) connection legs are established for a service.

When distributing the service control into three separate instances, the following properties are gained:

- optimal use of network resources controlled by network provider,

- network resource usage can be negotiated dynamically,

- reusability of terminal software (service specific modules offered by the VOD server),

- QoS can be defined by user or by service provider,

- each entity can have its own security management,

- user profile configuration can be managed locally and

- service profile management can be implemented in the VOD server.

This distributed alternative seems to be flexible enough to be used for networked applications. The distribution of the control gives the freedom to configure instance specific matters by each stakeholder. The architecture gives the modular solution and the modules can be reused for new services.

The solution creates requirements for the signalling. The protocols have to support dirstributed computing and control passing. Therefore the proposed protocol has to be analysed, how and if this distribution can be handled. This work is extensive and needs a lot of research of service examples. We shall only give some outlines here.

If the extension of DSS2 signalling is utilised the straightforward approach is to specify DSS2 supplementary services extension. This can be adopted from N-ISDN specification Q.932 facility messages. The question is, if this message set offers enough features to implement efficient call and charging control.

The other possibility is to have separate protocol and this can be approached from IN technology. The ITU-T Q.1214 and Q.1218 Recommendations specify the CS-1 protocols. Their broadband extensions would be needed and these extensions have been discussed to be added into IN Capability Set 3.

It is important to remember that control distribution extends requirements for both DSS and network control signalling. An important issue which may affect a great deal on how to select architecture is the different roles of service provider and network provider. Furthermore an important open issue is how to arrange broadband network charging. Is it completely a task of network providers or is it divided between service providers and network providers?

\section{CONCLUDING EXAMPLE}

Let us finally present a VOD service with signalling and distributed service logic according to the scenarios given in Figures 5-5 and 5-6. The organizational roles within the VOD service are Service Subscriber (SS), Network Operator (NO), Concept Provider (CP) and Service Provider (SP). We believe that these roles are substantial for broadband VANS and Media services. In present media terminology we can say that the CP acts like a newspaper publisher and the SP acts like an advertiser or news editor. These different types of stakeholders should have different privileges to control the services. The $\mathrm{CP}$ should have almost similar rights as the network operator in network and service control, the control of the capacity allocation and charging should be allowed. The CP needs to utilize service control interfaces with NO and SP. The SP should control communication with SS and furthermore SP should be able to 
deliver assisting information to $\mathrm{CP}$, so that more capacity or new connection to next server (in a pool) can be requested. The INAP type external control is used by the Concept Provider's Service Node (CP-SN) and the extension of the Q.2931 control is used by the Service Provider's Service Nodes (SP-SN1).

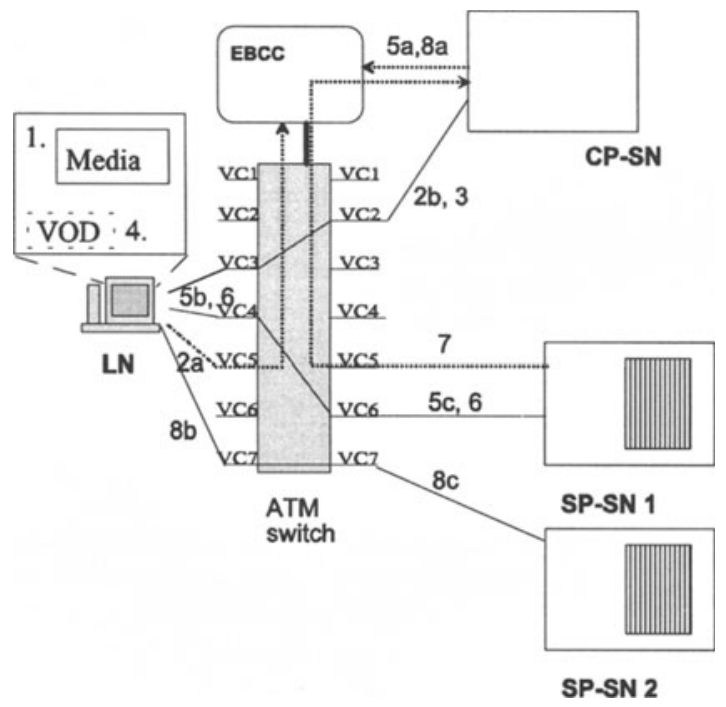

Figure 6-1. The VOD service example with distributed applications.

The Concept Provider's Service Node controls combinations of several services from different Service Provider's Service Nodes. Since the control of services is in the CP-SN, the interfaces, access rights and service parameters can be managed. CP-SN could also be an environment to manage the feature interactions of the services.

The service invocation and use sequence could be the following:

1. initial Service Icon is in $\mathbf{L N}$,

2. icon click activates the connection to CP Service Node (CP-SN),

3. a new icon palette is given to $\mathrm{LN}$,

4. LN activates a service icon,

5. the connection to SP Service Node (SP-SN1) is created,

6. the service is accessed and controlled via control channel between LN and SP-SN1,

7. SP-SN1 requests new network resource,

8. LN connection is rerouted by CP-SN to new destination (SP-SN2) and finally

9. the service is terminated.

This service logic distribution was presented first in [Wint]. The Icon concept as the service stub in the Local Node and the Service Palette concept as the service directory and control library in the Concept Provider Service Node acting as SCP are presented in [BINP]. In that study the ATM switch which supports this kind of Extended Basic Call Control was 
called as B-SSP and the Concept Provider Service Node was called as B-SCP. The synergic combinations of services provided by the Concept Provider were called Service Palettes.

\section{REFERENCES}

[N7524] ISO/IEC JTC1/SC21 N7524, ISO Committee Draft on Basic reference Model of Open Distributed Processing - Part 2: Descriptive Model, 1992

[Q931] CCITT Recommendation Q.931, ISDN User-Network Interface Layer 3 Specificatiom for Basic Call Control (DSS1)

[Q932] CCITT Recommendation Q.932, Generic Procedures for the Control of ISDN Supplementary Services

[Q12XY] CCITT Recommendation Series Q.1200 on Intelligent Networks

[Q2931] ITU-T Recommendation Q.2931, B-ISDN, Digital Subscriber Signalling No. 2 (DSS2), User Network Interface Layer 3, Specification for Basic Call Connection Control

[R1093] RACE Project R.1093 (ROSA) Deliverable 93/BTL/DNR/DS/A/005/b1, The RACE Architecture, Rel. Two, Version 2, RACE, 1992

[X722] CCITT Recommendation X.722, Guidelines for the Definition of managed Objects (GDMO)

[X725] CCITT Recommendation X.725, General Relationship Model

[Bellc] Bellcore SR-NWT-002268, Cycle 1 Specification for Information Networking Architecture (INA), Issue 2, 1992

[BINP] K. Molin, O. Martikainen, Broadband Intelligent Network Project, Workshop on Intelligent Networks, Lappeenranta University of Technology, August 1994

[Forum] ATM Forum, Introduction to ATM, The ATM Forum, 1993

[OMT] J.Rumbaugh, M.Blaha, W.Premerlani, F.Eddy and W.Lorensen, Object-Oriented Modeling and Design, Prentice Hall, N.J., 1991

[TINA] P. Leydekkers, V. Gay, Multimedia Services in TINA and RM-ODP, IN'94 Workshop, Heidelberg, 24-26.5.1994

[ValC] O. Martikainen, A. Lahti, Value Creation in Networks, Helsinki School of Economics, Report W-67, February 1994

[Wint] K. Molin, O. Martikainen, Intelligent Network Tutorial, The Second Winterschool on Telecommunications, Helsinki, Telecom Finland, March 1994 